\title{
How I Treat Metastatic Hormone-Sensitive Prostate Cancer?
}

\author{
Deepak Dabkara $\quad$ Debapriya Mondal ${ }^{1} \quad$ Joydeep Ghosh ${ }^{1} \quad$ Bivas Biswas $^{1} \quad$ Sandip Ganguly ${ }^{1}$ \\ 1Department of Medical Oncology, Tata Medical Center, Kolkata, \\ Address for correspondence Deepak Dabkara, MD, DM, \\ West Bengal, India \\ Action Area I, Newtown, Kolkata, West Bengal, 700160, India \\ (e-mail: deepakdabkara@yahoo.com).
}

Int J Med Paediatr Oncol 2021;42:100-107.

\begin{abstract}
Keywords

- hormone-sensitive

- metastatic

- prostate

Androgen deprivation therapy (ADT) combined with docetaxel or antiandrogens (abiraterone, enzalutamide, or apalutamide) improved the outcomes in men with metastatic hormone-sensitive prostate cancer (mHSPC). When multiple options are available, the dilemma remains how to choose among these options. Similarly, issues of bone health, long-term side effects of therapies, and hereditary risk need to be discussed for comprehensive care. In the present article, we reviewed the relevant evidence for the treatment of mHSPC. ADT alone is not the current standard of care for most patients. In these times of plenty and price crisis, it is imperative to find the best option for treating these patients.
\end{abstract}

\section{Introduction}

In a proportion of prostate cancer patients, there is evidence of metastatic disease at diagnosis or the disease recurs as distant metastasis despite standard curative treatment. An Indian hospital-based study reported that $70 \%$ of prostate cancer patients had metastasis at diagnosis. ${ }^{1}$ The upfront management of men with metastatic hormone-sensitive prostate cancer ( $\mathrm{mHSPC}$ ) had been with only androgen deprivation therapy (ADT), for a long time, either with medical or surgical castration. ${ }^{2}$ However, this scenario has recently changed after the publication of phase III randomized control trials that combine other agents with ADT upfront and clinicians now have various options to choose from. Each of these agents such as docetaxel, abiraterone acetate, enzalutamide, and apalutamide combined with ADT has shown significant survival benefit over ADT in randomized clinical trials. There is a lack of direct evidence to suggest the best choice as none of the trials include a head-on comparison between the available options. Factors such as toxicity profile, cost, and physicians' and patients' preferences are vital in choosing one of these options over the other. In the present manuscript, we

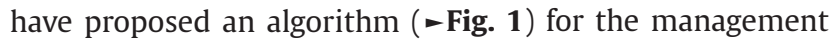
of patients with mHSPC from the currently available, most relevant literature on the topic.

\section{Androgen Deprivation Therapy}

ADT can be offered in the form of bilateral orchiectomy (surgical castration) or medical castration. Bilateral orchiectomy may be appropriate when a rapid decline in testosterone is needed (e.g., worsening obstructive urinary symptoms and imminent cord compression) or when cost or compliance to medical castration is a concern. In medical castration with gonadotropin-releasing hormone $(\mathrm{GnRH})$ agonists, a flare in serum testosterone may result from an initial short-term surge of luteinizing hormone (LH), worsening the symptoms. Antiandrogens (e.g., flutamide and bicalutamide) used for 2 to 4 weeks may be effective in preventing this flare phenomenon. ${ }^{3,4} \mathrm{GnRH}$ antagonist degarelix is not associated with this surge of $\mathrm{LH}$ and can be a substitute to a GnRH
DOI https://doi.org/ $10.1055 / \mathrm{s}-0041-1729725$ ISSN 0971-5851
(C)2021. Indian Society of Medical and Paediatric Oncology.

This is an open access article published by Thieme under the terms of the Creative Commons Attribution-NonDerivative-NonCommercial-License, permitting copying and reproduction so long as the original work is given appropriate credit. Contents may not be used for commercial purposes, or adapted, remixed, transformed or built upon. (https://creativecommons.org/licenses/by-nc-nd/4.0/).

Thieme Medical and Scientific Publishers Pvt. Ltd. A-12, 2nd Floor, Sector 2, Noida-201301 UP, India 


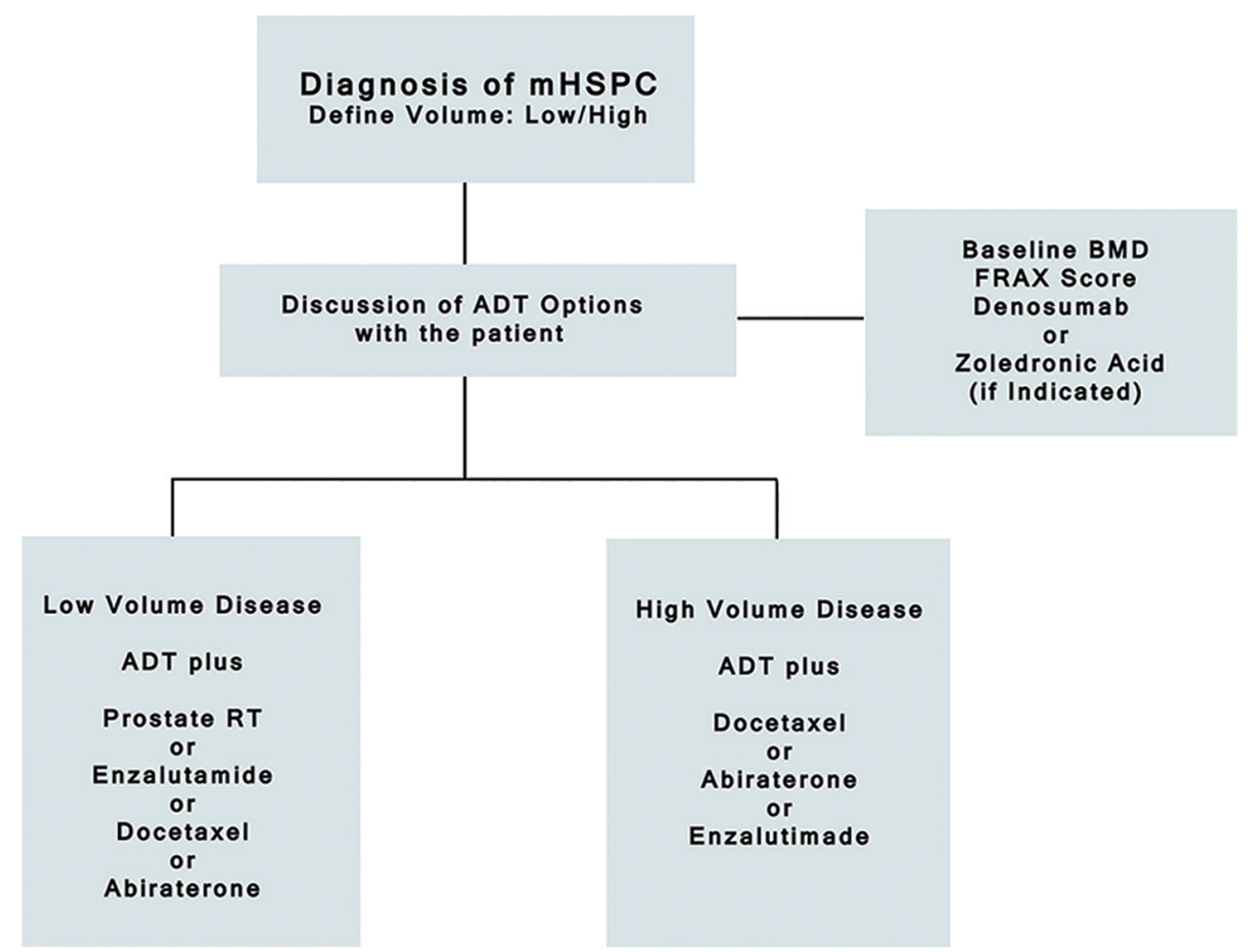

Fig. 1 Treatment algorithm. ADT, androgen deprivation therapy; BMD, bone mineral density; mHSPC, metastatic hormone-sensitive prostate cancer; RT, radiotherapy.

agonist when a rapid fall in serum testosterone is necessary. Incidence of severe cardiac adverse events may be lower with the use of degarelix compared with GnRH agonists, ${ }^{5}$ an ongoing prospective trial is comparing the risk of cardiovascular events with the use of GnRH agonists and antagonists. ${ }^{6}$ The newer oral agent relugolix that has shown better testosterone suppression and cardiovascular safety profile in a phase III randomized trial compared with leuprolide is another potential option for ADT. ${ }^{7}$

ADT has several side effects that can diminish the quality of life, which include loss of lean body mass, obesity, sexual dysfunction, vasomotor instability, gynecomastia, fatigue, cardiovascular, and metabolic abnormalities. Another concerning side effect is bone demineralization, which can lead to osteoporotic fractures. ${ }^{8,9}$ Intermittent ADT is a strategy to reduce these adverse effects by pausing ADT when patients have responded to treatment and restarting at progression. A phase III intergroup trial did not find intermittent ADT noninferior to continuous ADT in respect of overall survival (OS). ${ }^{10}$ In spite of the theoretical advantage, intermittent ADT is not recommended for patients of MHSPC save for those with an elevated serum prostate-specific antigen (PSA) as the sole manifestation of disseminated prostate cancer, ${ }^{11}$ unless quality of life is the main expectation.

\section{Docetaxel}

The first evidence of benefit from a combination treatment was with docetaxel that had previously improved OS in patients of metastatic castration-resistant prostate cancer (mCRPC). ${ }^{12,13}$ The CHAARTED trial that accrued 790 patients with hormone-naive metastatic prostate cancer reported improved survival of patients with MHSPC when docetaxel was added to ADT compared with ADT alone. ${ }^{14}$ However, in a smaller study (GETUG-AFU15), docetaxel plus ADT did not improve survival in patients of mHSPC and reported more toxicities. ${ }^{15}$ This conflicting evidence was explained by the difference in the burden of disease between the patient populations enrolled in these studies; they also differed in the number of chemotherapy cycles used. In the CHAARTED study, the majority (65\%) of the patients had high-volume disease (-Table 1). Those with high-volume disease had a significant OS benefit from adding docetaxel to ADT (median OS 51.2 months vs. 34.4 months, hazard ratio $[\mathrm{HR}]=0.63$; 95\% confidence interval $[\mathrm{CI}]: 0.50-0.79 ; p<0.001$ ), but this was not evident in patients with low-volume disease (HR: $1.04 ; 95 \%$ CI: $0.70-1.55 ; p=0.86) .{ }^{17}$ The arm C of the multiarm, multistage platform, STAMPEDE trial showed ${ }^{18}$ a significant OS benefit for patients treated with the combination of docetaxel and ADT (median OS 60 months in ADT plus docetaxel vs. 45 months in ADT only [HR: 0.76 ; $95 \% \mathrm{CI}$ :

Table 1 Stratification by metastatic disease burden

\begin{tabular}{|l|l|}
\hline Trial & Definition \\
\hline CHAARTED $^{14}$ & $\begin{array}{l}\text { Defined as: (i) four or more bone metastases on } \\
\text { high volume bone scan, including one or more } \\
\text { outside the vertebral bodies or pelvis, and/or } \\
\text { (ii) visceral metastases }\end{array}$ \\
\hline LATITUDE $^{16}$ & $\begin{array}{l}\text { Defined as meeting at least two of three high risk } \\
\text { criteria: (i) Gleason score }>8 \text {, (ii) presence of } \geq 3 \\
\text { lesions on bone scan, (iii) presence of measurable } \\
\text { visceral lesions }\end{array}$ \\
\hline
\end{tabular}


0.62-0.92]). Long-term survival results from the STAMPEDE trial reported OS benefit for the combination of docetaxel with ADT in mHSPC (59.1 vs. 43.1 months, stratified log-rank test $p=0.003$, HR: $0.81 ; 95 \% \mathrm{CI}: 0.69-0.95)$ irrespective of metastatic disease burden. Improvements in OS, time to metastatic progression, and time to next treatment for both low- and high-burden metastatic disease were reported in the updated analysis. ${ }^{19} \mathrm{~A}$ meta-analysis on the data from the aforementioned trials reported survival benefit in patients treated with docetaxel plus ADT (HR: 0.77; 95\% CI: 0.68-0.87 $[p<0.001]$ ), reaffirming upfront use of chemotherapy in mHSPC. ${ }^{16}$

However, patients receiving combination treatment with docetaxel and ADT had higher rates of neutropenia, febrile neutropenia, and fatigue. Febrile neutropenia rates among patients were $16 \%$ in GETUG-AFU $15,13 \%$ in STAMPEDE, and $6 \%$ in CHAARTED trial. ${ }^{20}$

\section{Abiraterone Acetate}

Abiraterone acetate, an irreversible inhibitor of CYP17A1 which initially got approval for the treatment of MCRPC, ${ }^{21,22}$ reported OS benefit in MHSPC when added to ADT in two phase III trials, LATITUDE and STAMPEDE. ${ }^{23,24}$

The LATITUDE trial enrolled 1199 patients with treatment-naive mHSPC with two or more high-risk features ( - Table 1) and compared ADT plus abiraterone with ADT alone and reported significantly better survival (HR: 0.66 [95\% CI: 0.56-0.78]; $p$ < 0.0001) in the abiraterone with ADT arm. Risk of radiographic progression was reduced by $53 \%$ with the addition of abiraterone (HR: 0.47 [95\% CI: 0.39-0.55]; $p<0.001$ ). Abiraterone significantly prolonged time to pain progression, next antineoplastic treatment, the start of cytotoxic therapy, PSA elevation $(p<0.001$ for all comparisons), and next symptomatic skeletal event ( $p=$ 0.009). Most subgroups benefitted with abiraterone added to ADT with the possible exceptions of the patients with Eastern Cooperative Oncology Group performance status 2, age of 75 years or more, or with a Gleason score of $<8$. In a retrospective observational study from an Indian tertiary care cancer center, treatment outcomes were comparable to phase III studies in patients treated with ADT combined with abiraterone. $^{25}$

The STAMPEDE trial that randomized 1917 patients with HSPC (52\% metastatic) to receive abiraterone acetate with ADT (arm G) or ADT alone reported a 39\% reduced death risk (HR: $0.61 ; 95 \% \mathrm{CI}: 0.49-0.75$ ) in patients with mHSPC. Further, the risk of treatment failure was also significantly reduced in patients receiving abiraterone (HR: 0.29 (HR: $0.29,95 \%$ CI: 0.25-0.34); $p<0.001$ ). Interestingly, a post hoc subgroup analysis of OS in metastatic patients included in STAMPEDE found survival benefit with coadministration of abiraterone and ADT irrespective of risk (LATITUDE criteria) or volume (CHAARTED criteria); although it required four times the number of high-risk patients to be treated to find the OS benefit in the low-risk group. ${ }^{26}$

A prespecified direct comparison between the abiraterone and docetaxel arms of STAMPEDE did not show a difference in OS, prostate cancer-specific survival, and symptomatic skeletal events. Frequency of Grade 3 and 4 toxicities was comparable in both arms but comprised different toxicities. Neutropenia of all grades and febrile neutropenia occurred more frequently with docetaxel, while endocrine, cardiovascular, hepatic disorders, and hypokalemia were more common with abiraterone. ${ }^{27}$

\section{Novel Androgen Receptor Antagonists}

Enzalutamide, a second-generation androgen receptor inhibitor initially approved for the treatment of CRPC, ${ }^{28,29}$ has been explored as a treatment option combined with ADT in the mHSPC setting in two phase III trials. ${ }^{30,31}$

Interim analysis of the ENZAMET trial showed a significant OS benefit in patients treated with enzalutamide compared with those who received only ADT (HR $=0.67, p=0.002)$. In the ARCHES trial, median radiographic progression-free survival (rPFS) for the placebo plus ADT arm was 19.4 months (95\% CI: 16.6, NR, not reached), whereas median rPFS was not reached for the enzalutamide arm (HR: 0.39; 95\% CI: 0.30 , $0.50 ; p<0.0001)$. The enzalutamide arm also had a statistically significant improvement in time to initiate a new systemic treatment (HR: 0.28; 95\% CI: 0.20, 0.40; $p<0.0001$ ). On December 16, 2019, the US Food and Drug Administration (FDA) approved enzalutamide for patients with mHSPC.

In the TITAN trial, ${ }^{32}$ a benefit for apalutamide was shown for men with both high-volume and low-volume metastatic disease. There was a significant improvement in rPFS (HR: 0.48; 95\% CI: 0.39-0.60), and the risk of radiographic progression or death was reduced by $52 \%$. In the apalutamide arm, the median rPFS was not reached and the same was 22.1 months in the placebo arm. Importantly, apalutamide showed a significant improvement in OS (HR: 0.67; 95\% CI: 0.51-0.89), and reduced the risk of death by $33 \%$. Median OS was not reached for either apalutamide or placebo groups. Apalutamide was approved for the treatment of mHSPC in the United States in September 2019.

In randomized trials, $0.5 \%$ of the patients receiving enzalutamide experienced seizures, while fall and fractures, ischemic heart disease, and posterior reversible encephalopathy syndrome remain other concerning side effects. ${ }^{33}$ In the TITAN trial, $4 \%$ of the patients receiving apalutamide had ischemic cardiac events. Apalutamide has also been associated with falls, fractures, and cardiac events in clinical trial experience. $^{34}$

\section{Bone Health}

Long-term ADT has been shown to adversely affect bone mineral density (BMD) and raise pathological fracture risk in men. A large observational study found that prostate cancer patients have significantly higher fracture risk when they receive ADT (19.4 vs. $12.6 \% ; p<0.001) .{ }^{35}$ Metastatic prostate cancer patients have bone involvement in an estimated $90 \%$ of cases, ${ }^{36}$ and evidence suggests that men with metastatic prostate cancer have a higher incidence of osteopenia and osteoporosis compared with age-matched 
control populations even prior to starting ADT. ${ }^{37}$ The National Institute for Health and Care Excellence UK guidelines recommend evaluation of fracture risk for all men receiving ADT and that those found to have osteoporosis should be offered treatment. ${ }^{38}$ The European Association of Urology, European Society for Radiotherapy (RT) and Oncology and International Society for Geriatric Oncology guidelines recommend BMD assessment prior to starting long-term ADT. ${ }^{39}$ In noncancer populations, $\mathrm{FRAX}^{40}$ a fracture risk assessment tool, is commonly used to calculate the 10 -year probability of major osteoporotic fracture (spine/ hip/forearm/humeral fractures) to warrant the need for BMD assessment and/or treatment. The FRAX algorithm is useful in choosing metastatic prostate cancer patients who require early bone-directed therapy. ${ }^{41}$ However, there remains controversy regarding the benefit of using bone-directed therapy in men with mHSPC as compared with mCRPC. The STAMPEDE trial, in which high risk prostate cancer patients with and without osseous metastases were enrolled, the addition of zoledronic acid (ZA) to standard care failed to improve OS, while the addition of ZA to the arm that combined docetaxel to standard care did not show any advantage in failure free survival, skeletal related events (SREs), or OS. In the CALGB 90202, the use of ZA for HSPC was not associated with a decrease in SRE risk compared with treatment initiation after progression to CRPC. ${ }^{42}$

Evidence with the use of denosumab, a fully human monoclonal antibody immunoglobulin G 2 against Receptor activator of nuclear factor kappa-B ligand (RANK-L), is inadequate in MHSPC compared with MCRPC where its role is established. ${ }^{43-45}$ Denosumab is FDA approved for the prevention of bone loss and fractures during ADT based on a phase III study in patients with nonmetastatic prostate cancer receiving $A D T$, where denosumab improved BMD by $6.7 \%$ and reduced fracture risk (1.5 vs. $3.9 \%$ ) compared with placebo. ${ }^{46}$

In view of lack of evidence to support the use of bisphosphonates or denosumab in combination with androgen receptor targeted agents or chemotherapy in patients with $\mathrm{mHSPC}$, routine use of bone-directed therapy is not recommended, but in men with osteoporosis or higher fracture risk detected prior to initiating ADT, such treatment should be considered.

The National Osteoporosis Foundation guidelines ${ }^{47}$ recommended daily calcium (1,000-1,200 mg) and Vitamin D3 (400-1,000 IU) supplementation along with additional treatment for men aged 50 years or older with osteopenia (T-score between 1.0 and 2.5 at the femoral neck, total hip, or lumbar spine by dual-energy X-ray absorptiometry [DEXA] scan) and a 10-year probability of hip fracture $>3 \%$ or a 10-year probability of a major osteoporosis-related fracture $>20 \%$. In our practice, we initiate treatment with ZA (5 mg IV annually) or with denosumab (60 mg subcutaneous every 6 months) when the fracture risk derived from BMD and FRAX score (risk of hip fracture $>3 \%$, or risk of major osteoporotic fracture $>20 \%$ ) necessitates drug therapy. A baseline DEXA scan before the start of therapy and another after 1 year of therapy is recommended to monitor treatment response. ${ }^{48}$

\section{Role of Radiotherapy}

The benefit of local therapy in conjunction with ADT for those presenting with $\mathrm{mHSPC}$ has been a subject of debate. The role of local RT concurrent with ADT has been tested in two randomized trials; among them, the phase III HORRAD trial randomized 432 men with primary metastatic prostate cancer with bone metastases and a serum PSA $>20 \mathrm{ng} / \mathrm{mL}$ to ADT with or without external beam RT. ${ }^{49}$ At a median follow-up of 47 months, median OS did not improve from the addition of RT (45 vs. 43 months, HR: 0.90; 95\% CI: 0.70-1.14); however, the addition of RT prolonged the median time to PSA progression (median: 15 vs. 12 months, HR: 0.78; 95\% CI: 0.63-0.97). An unplanned subgroup analysis suggested that men with fewer than five metastases might have survival benefits (HR: 0.68; 95\% Cl: $0.42-1.10$ ) when treated with RT in conjunction with ADT.

Survival benefit for RT to the prostate for unselected men with newly diagnosed metastatic prostate cancer was also not demonstrated in the phase III STAMPEDE trial, ${ }^{50}$ but there was an improvement in failure-free survival (3-year failure-free survival 32 vs. 23\%, HR: 0.76; 95\% CI: 0.68-0.84). In a prespecified subgroup analysis, OS benefit was seen with RT in the men with a low metastatic burden (CHAARTED definition) at diagnosis (3-year survival 81 vs. 73\%, HR for death $0.68,95 \% \mathrm{CI}: 0.52-0.90)$ but not in those with a high metastatic burden (HR: 1.07; 95\% CI: 0.90-1.28).

Pooled results of both trials found $\sim 7 \%$ improvement in survival in men with fewer than five bone metastases, along with an overall improvement in biochemical progression-free survival (HR: 0.74; 95\% CI: 0.67-0.82) and failure-free survival (HR: 0.76; 95\% CI: 0.69-0.84). ${ }^{51}$

\section{Imaging and Assessment during Treatment}

In the initial evaluation of men diagnosed with conventional imaging as mHSPC at presentation, there may be some role of next-generation imaging (positron-emission tomography-computed tomography [PET/CT], PET/magnetic resonance imaging [MRI], and whole-body MRI) to clarify the burden of disease and this can help to choose either multimodality management of oligometastatic disease or systemic anticancer therapy alone, but prospective data to guide such decision are limited. ${ }^{52}$

Men with $\mathrm{mHSPC}$ who receive systemic therapy require periodic assessments to identify signs and symptoms of disease progression, as well as the side effects of treatment (-Table 2). Measurement of serum PSA at specific intervals is the mainstay of testing. The current National Comprehensive Cancer Network (NCCN) guideline recommends testing PSA every 3 to 6 months during treatment for metastatic prostate cancer. ${ }^{53}$ A rise in PSA values, or development of new symptoms, is the cue for a radiologic assessment. When PSA levels do not decline in response to therapy or rise, the adequacy of castrate status (defined as serum testosterone $<50 \mathrm{ng} / \mathrm{mL}$ ) should be checked. ${ }^{54}$ 
Table 2 Drugs and periodic monitoring

\begin{tabular}{|l|l|}
\hline Drug & Periodic monitoring \\
\hline Docetaxel & CBC and differential, LFT before each cycle \\
\hline $\begin{array}{l}\text { Abiraterone ace- } \\
\text { tate+prednisolone }\end{array}$ & $\begin{array}{l}\text { Baseline: CBC and differential, LFT, } \\
\text { creatinine, glucose, electrolytes. For the } \\
\text { first three cycles: monitor blood pressure, } \\
\text { serum potassium, LFT, every 2 weeks. } \\
\text { Before each outpatient visit (every 4 } \\
\text { weeks): CBC and differential, LFT, creati- } \\
\text { nine, glucose, electrolytes, regular moni- } \\
\text { toring of BP }\end{array}$ \\
\hline Enzalutamide & $\begin{array}{l}\text { Baseline: CBC and differential, creatinine, } \\
\text { electrolytes, blood pressure, ECG } \\
\text { Before each outpatient visit: blood pres- } \\
\text { sure, serum creatinine, electrolytes, ECG }\end{array}$ \\
\hline
\end{tabular}

Abbreviations: BP, blood pressure; CBC, complete blood count; ECG, electrocardiogram; LFT, liver function tests.

\section{Genetic Testing in Prostate Cancer}

It is important in prostate cancer to address the inherited component. A family history of prostate cancer and/or other cancers (e.g., breast cancer diagnosed at an age younger than 50 years, male breast cancer, ovarian cancer, colorectal cancer, pancreatic cancer, and melanoma) may be associated with an increased risk of heritable prostate cancer. The St. Gallen Advanced Prostate Cancer Consensus Conference $2017^{55}$ and the Philadelphia Prostate Cancer Consensus Conference $2019^{56}$ have addressed the issues of genetic counseling and genetic testing for prostate cancer. The Philadelphia guidelines recommend genetic testing for all men with metastatic prostate cancer and for those with a family history of cancer with gene panels wherever feasible, which should include BRCA1/2 and DNA mismatch repair genes. The NCCN prostate cancer guidelines ${ }^{53}$ recommend $^{-}$ offering genetic testing to men with a personal history of high- or very high-risk regional or metastatic prostate cancer, or localized disease with intraductal histology, as well as a family history of high-risk germline mutations (e.g., BRCA1 and BRCA2, Lynch mutation, Ashkenazi Jewish ancestry), or to those with a strong family history of cancer.
Clinical trials of poly-adenosine diphosphate-ribose polymerase inhibitors have shown promising responses in men with germline or somatic mutations in BRCA2, BRCA1, ATM, CHEK2, PALB2, and in other homologous recombination DNA repair genes. ${ }^{57,58}$ Olaparib and rucaparib have been approved by the FDA for men with mCRPC harboring these mutations. On the other hand, advanced prostate cancer patients with loss of DNA mismatch repair may benefit from treatment with immune checkpoint inhibitors. However, these genetic components do not currently have any implications for the treatment of mHSPC.

\section{How to Choose One Option}

The choice between chemohormonal therapy and combined androgen receptor-targeted therapy (abiraterone or enzalutamide or apalutamide) is difficult, owing to the similar efficacy outcomes ( - Table 3 ) in cross-trial comparisons. Differences (-Table 4 ) in toxicities and costs rather than the subtle differences in efficacy end points might, at times, guide selection among the many choices approved in the first-line treatment of mHSPC. Docetaxel appears to be the most cost-effective and efficient approach in combination with ADT for men with high-volume MHSPC; it is given for a relatively shorter period of time- 18 weeks with reversible albeit severe short-term chemotherapy toxicities. However, in men with low-volume disease, the evidence of benefit from adding docetaxel has been conflicting. Unfortunately, similar concerns with evidence of clear benefit in low-volume disease remain with abiraterone as well since its indication in low-volume disease is based on a post hoc analysis of STAMPEDE that was not powered to find an OS benefit for the low-risk population. A cost-effectiveness analysis from the US report docetaxel is a more cost-effective option than abiraterone in the treatment of $\mathrm{MHSPC} .{ }^{59}$ Abiraterone is recommended daily until disease progression with a median time on treatment of $\sim 33$ months and is expected to be costlier for our population. Patient preferences and comorbidities often help in the decision-making process. Docetaxel usually is preferred for patients who wish for a shorter treatment time.

Table 3 Comparison of overall survival of trials (not head-to-head) in first-line treatment of metastatic hormone-sensitive prostate cancer

\begin{tabular}{|c|c|c|c|c|c|c|}
\hline \multirow{2}{*}{$\begin{array}{l}\text { Agent } \\
\text { Study }\end{array}$} & \multicolumn{2}{|l|}{ Docetaxel } & \multicolumn{2}{|l|}{ Abiraterone } & \multirow{2}{*}{$\begin{array}{l}\text { Enzalutamide } \\
\text { ENZAMET }^{30}\end{array}$} & \multirow{2}{*}{$\begin{array}{l}\text { Apalutamide } \\
\text { TITAN }^{32}\end{array}$} \\
\hline & CHAARTED ${ }^{14}$ & $\begin{array}{l}\text { STAMPEDE }^{18,19} \\
\text { Arm C M1 patients }\end{array}$ & LATITUDE $^{23}$ & $\begin{array}{l}\text { STAMPEDE }{ }^{4} \\
\text { Arm G M1 } \\
\text { patients }\end{array}$ & & \\
\hline \multirow[t]{3}{*}{ OS } & $\begin{array}{l}57.6 \text { vs. } 47.2 \\
\text { months }\end{array}$ & 59.1 vs. 43.1 months & $\begin{array}{l}53.3 \text { vs. } 36.5 \\
\text { months }\end{array}$ & $0.61(0.49-0.75)$ & $0.67(0.52-0.86)$ & $0.67(0.51-0.89)$ \\
\hline & $0.72(0.59-0.89)$ & $0.76(0.62-0.92)$ & $0.66(0.56-0.78)$ & - & - & - \\
\hline & 10.4 months & 16 months & 15 months & & & \\
\hline OS-HV & $0.63(0.50-0.79)$ & $0.81(0.64-1.02)$ & $0.62(0.52-0.78)$ & $0.60(0.46-0.78)$ & $0.80(0.59-1.07)$ & $0.68(0.50-0.92)$ \\
\hline OS-LV & $1.04(0.70-1.55)$ & $0.76(0.54-1.07$ & $0.72(0.47-1.10)$ & $0.64(0.42-0.97)$ & $0.43(0.26-0.72)$ & $0.67(0.34-1.32)$ \\
\hline
\end{tabular}

Abbreviations: HV, high volume; LV, low volume; mHSPC, metastatic hormone-sensitive prostate cancer; OS, overall survival.

Figures are hazard ratios with $95 \%$ confidence intervals from select phase III trials in the upfront treatment of mHSPC. 
Table 4 Comparison of unique factors and toxicities between upfront options

\begin{tabular}{|c|c|c|c|}
\hline & Docetaxel & Abiraterone & Enzalutamide \\
\hline $\begin{array}{l}\text { Length of } \\
\text { treatment }\end{array}$ & Shorter & Longer & Longer \\
\hline \multirow[t]{2}{*}{ Finances } & $\begin{array}{l}\text { Relatively inexpensive, likely to be } \\
\text { covered by insurance }\end{array}$ & Costlier, likely to be out of pocket & Costlier, likely to be out of pocket \\
\hline & $\begin{array}{l}\text { Rs. } 10,000-15,000 \text { per cycle for } \\
6 \text { cycles }\end{array}$ & Rs. 10,000 and above per month & Rs. 20,000 and above per month \\
\hline Salient toxicities & $\begin{array}{l}\text { Neutropenia, peripheral neuropa- } \\
\text { thy, alopecia }\end{array}$ & $\begin{array}{l}\text { Liver enzyme elevation, hypo- } \\
\text { kalemia, hypertension }\end{array}$ & $\begin{array}{l}\text { CNS-seizure, PRES, cognitive, falls, and } \\
\text { fractures }\end{array}$ \\
\hline Disease burden & High & High & Any \\
\hline Steroid & Not required & Required & Not required \\
\hline
\end{tabular}

Abbreviations: CNS, central nervous system; PRES, posterior reversible encephalopathy syndrome.

Patients with preexisting hypertension, hepatic derangement, and metabolic abnormalities may not be suitable candidates for abiraterone. Further, there is nothing to suggest that abiraterone offers any benefit in the population considered ineligible for docetaxel-those with poor performance status and the elderly, as a benefit in these subgroups was unclear in the LATITUDE trial. Enzalutamide, the new entrant in this setting, has proven to be effective for both low- and high-volume disease and is an attractive option for those wishing to avoid chemotherapy and steroids. The unique adverse events with enzalutamide such as falls, seizures, syncope, cognitive, and mental impairment warrant caution while selecting patients. In general, patients who wish to avoid chemotherapy at all costs and wish to minimize hospital visits should be offered an androgen receptor-targeted therapy. In absence of prospective evidence, the question of whether we should add docetaxel or abiraterone to ADT in patients with low-volume or low-risk mHSPC remains a difficult one and such treatments should be offered with caution. Patients of MHSPC with a low burden of bone metastases (four or fewer bone metastases, with none outside the vertebral bodies or pelvis) and no visceral metastases may be offered RT to the prostate in conjunction with systemic therapy. Finally, there are groups of patients who may do well with ADT alone, even intermittently, sometimes for many years. The ultimate choice for an individual patient largely depends on the oncologist elaborating risks and benefits of each available option while considering the patient's comorbid conditions, access to treatment, financial aspects, and preference.

\section{What I Follow in My Practice}

Most of the patients do present to our center with PSA, histopathology, and some imaging (mainly MRI pelvis and bone scan). I generally review histopathology at our center as per the institutional policy unless the report is from a reputed cancer center or oncopathologist. If the available imaging already suggests metastatic disease, I do not advise ${ }^{68}$
Ga prostate-specific membrane antigen (PSMA) PET/CT I use CT scans of the thorax, abdomen, pelvis, and bone scan to assess metastatic disease volume. For patients who present with PSA rise after past curative treatment, I prefer PSMA PET/CT scan. I discuss medical and surgical castration with my patients and the cost of treatment is often the main deciding factor between the two. ${ }^{60}$ I recommend a baseline BMD before starting ADT. If the FRAX score is suggestive of a high risk of fracture, they are offered ZA $5 \mathrm{mg}$ once a year. For high-volume disease, I discuss with the patients all three options (ADT with docetaxel or abiraterone or enzalutamide). For patients with low-volume disease, I discuss ADT and local RT, and ADT with one among enzalutamide, docetaxel, or abiraterone. I follow current genetic testing guidelines in prostate cancer and discuss germline mutation testing for all patients of mHSPC.

\section{Conclusion}

The oncologist's armamentarium for treating mHSPC is rapidly expanding as newer evidence demonstrates that combination therapy with one among docetaxel, abiraterone acetate, enzalutamide, or apalutamide provides a significant OS benefit when compared with ADT alone. The availability of many options with unique toxicity profiles allows oncologists flexibility in choosing the right option for individual patients. We await the results of ongoing randomized studies of darolutamide ${ }^{61}$ or further intensification of treatment ${ }^{62,63}$ to provide further guidance for clinicians. For now, it is rational to conclude that upfront combination approaches are the new standard of care for men with MHSPC, and some patients with low volume disease may benefit from the addition of RT, while ADT alone remains an option only in patients who are either not fit for the combination options or have unacceptable toxicities.

\section{Financial Support and Sponsorship}

Nil. 


\section{Conflicts of Interest}

There are no conflicts of interest.

\section{References}

1 Balasubramaniam G, Talole S, Mahantshetty U, Saoba S, Shrivastava S. Prostate cancer: a hospital-based survival study from Mumbai, India. Asian Pac J Cancer Prev 2013;14(4):2595-2598

2 Tangen CM, Hussain MH, Higano CS, et al. Improved overall survival trends of men with newly diagnosed M1 prostate cancer: a SWOG phase III trial experience (S8494, S8894 and S9346) J Urol 2012;188(4):1164-1169

3 Labrie F, Dupont A, Belanger A, Lachance R. Flutamide eliminates the risk of disease flare in prostatic cancer patients treated with a luteinizing hormone-releasing hormone agonist. J Urol 1987;138(4):804-806

4 Schulze H, Senge T. Influence of different types of antiandrogens on luteinizing hormone-releasing hormone analogue-induced testosterone surge in patients with metastatic carcinoma of the prostate. J Urol 1990;144(4):934-941

5 Sciarra A, Fasulo A, Ciardi A, et al. A meta-analysis and systematic review of randomized controlled trials with degarelix versus gonadotropin-releasing hormone agonists for advanced prostate cancer. Medicine (Baltimore) 2016;95(27):e3845

6 Melloni C, Slovin SF, Blemings A, et al. Cardiovascular safety of degarelix versus leuprolide for advanced prostate cancer: the PRONOUNCE trial study design. J Am Coll Cardiol CardioOncol 2020;2:70-81

7 Shore ND, Saad F, Cookson MS, et al. HERO Study Investigators. Oral relugolix for androgen-deprivation therapy in advanced prostate cancer. N Engl J Med 2020;382(23):2187-2196

8 Ahmadi H, Daneshmand S. Androgen deprivation therapy: evidence-based management of side effects. BJU Int 2013;111(4):543-548

9 Gaztañaga M, Crook J. Androgen deprivation therapy: minimizing exposure and mitigating side effects. J Natl Compr Canc Netw 2012;10(9):1088-1095, quiz 1088, 1096

10 Hussain M, Tangen CM, Berry DL, et al. Intermittent versus continuous androgen deprivation in prostate cancer. $\mathrm{N}$ Engl J Med 2013;368(14):1314-1325

11 Klotz L, O'Callaghan CJ, Ding K, et al. A phase III randomized trial comparing intermittent versus continuous androgen suppression for patients with PSA progression after radical therapy: NCIC CTG PR.7/SWOG JPR.7/CTSU JPR.7/UK Intercontinental Trial CRUKE/01/013. JCO 2011;29(7(Suppl):3

12 Tannock IF, de Wit R, Berry WR, et al. TAX 327 Investigators. Docetaxel plus prednisone or mitoxantrone plus prednisone for advanced prostate cancer. N Engl J Med 2004; 351(15):1502-1512

13 Petrylak DP, Tangen CM, Hussain MH, et al. Docetaxel and estramustine compared with mitoxantrone and prednisone for advanced refractory prostate cancer. N Engl J Med 2004;351(15):1513-1520

14 Sweeney CJ, Chen YH, Carducci M, et al. Chemohormonal therapy in metastatic hormone-sensitive prostate cancer. $\mathrm{N}$ Engl J Med 2015;373(8):737-746

15 Gravis G, Fizazi K, Joly F, et al. Androgen-deprivation therapy alone or with docetaxel in non-castrate metastatic prostate cancer (GETUG-AFU 15): a randomised, open-label, phase 3 trial. Lancet Oncol 2013;14(2):149-158

16 Vale CL, Fisher DJ, White IR, et al. What is the optimal systemic treatment of men with metastatic, hormone-naive prostate cancer? A STOPCAP systematic review and network meta-analysis. Ann Oncol 2018;29(5):1249-1257

17 Kyriakopoulos CE, Chen YH, Carducci MA, et al. Chemohormonal therapy in metastatic hormone-sensitive prostate cancer: long-term survival analysis of the randomized phase III E3805 CHAARTED trial. J Clin Oncol 2018;36(11):1080-1087

18 Addition of Docetaxel, Zoledronic Acid, or Both to First-Line Long-Term Hormone Therapy in Prostate Cancer (STAMPEDE): Survival Results from an Adaptive, Multiarm, Multistage, Platform Randomised Controlled Trial-The Lancet. Available from: https://www.thelancet.com/journals/lancet/article/ PIIS0140-6736 (15) 01037-5/fulltext. Accessed April 9, 2021

19 Addition of Docetaxel to Hormonal Therapy in Low- and High-Burden Metastatic Hormone Sensitive Prostate Cancer. Long-Term Survival Results from the STAMPEDE Trial | Annals of Oncology | Oxford Academic. Available from: https:/| academic.oup.com/annonc/article/30/12/1992/5574397. Accessed April 9, 2021

20 Schweizer MT, Gulati R, Mostaghel EA, et al. Docetaxel-related toxicity in metastatic hormone-sensitive and metastatic castration-resistant prostate cancer. Med Oncol 2016;33(7):77

21 Ryan CJ, Smith MR, de Bono JS, et al. COU-AA-302 Investigators. Abiraterone in metastatic prostate cancer without previous chemotherapy. N Engl J Med 2013;368(2):138-148

22 de Bono JS, Logothetis CJ, Molina A, et al. COU-AA-301 Investigators. Abiraterone and increased survival in metastatic prostate cancer. N Engl J Med 2011;364(21):1995-2005

23 Abiraterone Plus Prednisone in Metastatic. Castration-Sensitive Prostate Cancer | NEJM. Available from: https://www.nejm. org/doi/ full/10.1056/NEJMoal704174. Accessed April 9, 2021

24 Abiraterone for Prostate Cancer Not Previously Treated with Hormone Therapy | NEJM. Available from: https://www.nejm. org/doi/full/10.1056/NEJMoal702900. Accessed April 9, 2021

25 Rajput A, Hussain SM, Sonthwal N, et al. Clinicoradiological profile and treatment outcomes in prostate cancer at a tertiary care cancer center in India. Indian J Med Paediatr Oncol 2020;41:187-192

26 Hoyle AP, Ali A, James ND, et al. STAMPEDE Investigators. Abiraterone in "High-" and "Low-risk" metastatic hormone-sensitive prostate cancer. Eur Urol 2019;76(6):719-728

27 Adding Abiraterone or Docetaxel to Long-Term Hormone Therapy for Prostate Cancer: Directly Randomised Data from the STAMPEDE Multi-Arm, Multi-Stage Platform Protocol | Annals of Oncology | Oxford Academic. Available from: https:// academic.oup.com/annonc/article/29/5/1235/4910513. Accessed April 9, 2021

28 Hussain M, Fizazi K, Saad F, et al. Enzalutamide in Men with nonmetastatic, castration-resistant prostate cancer. N Engl J Med 2018;378(26):2465-2474

29 Penson DF, Armstrong AJ, Concepcion R, et al. Enzalutamide versus bicalutamide in castration-resistant prostate cancer: the STRIVE Trial. J Clin Oncol 2016;34(18):2098-2106

30 Davis ID, Martin AJ, Stockler MR, et al. ENZAMET Trial Investigators and the Australian and New Zealand Urogenital and Prostate Cancer Trials Group. Enzalutamide with standard first-line therapy in metastatic prostate cancer. N Engl J Med 2019;381(2):121-131

31 Armstrong AJ, Szmulewitz RZ, Petrylak DP, et al. ARCHES: a randomized, phase III study of androgen deprivation therapy with enzalutamide or placebo in men with metastatic hormone-sensitive prostate cancer. J Clin Oncol 2019;37(32):2974-2986

32 Chi KN, Agarwal N, Bjartell A, et al. TITAN Investigators Apalutamide for metastatic, castration-sensitive prostate cancer. N Engl J Med 2019;381(1):13-24

33 Astellas Pharma US. Inc., Enzalutamide. U.S. Food and Drug Administration; 2019. Available from: https://www.accessdata. fda.gov/drugsatfda_docs/label/2019/203415s0151bl.pdf. Accessed April 9, 2021

34 Janssen Pharmaceutical Companies. Apalutamide. U.S. Food and Drug Administration; 2019. Available from: https://www. 
accessdata.fda.gov/drugsatfda_docs/label/2019/210951s 0011bl.pdf. Accessed April 9, 2021

35 Shahinian VB, Kuo YF, Freeman JL, Goodwin JS. Risk of fracture after androgen deprivation for prostate cancer. N Engl J Med 2005;352(2):154-164

36 Bubendorf L, Schöpfer A, Wagner U, et al. Metastatic patterns of prostate cancer: an autopsy study of 1,589 patients. Hum Pathol 2000;31(5):578-583

37 Immediate Dual Energy X-ray Absorptiometry Reveals a High Incidence of Osteoporosis in Patients with Advanced Prostate Cancer before Hormonal Manipulation-Hussain2003-BJU International-Wiley Online Library. Available from: https:// onlinelibrary.wiley.com/doi/full/10.1046/j. 1464410X.2003.0447 1. x?sid=nlm\%3Apubmed. Accessed April 9, 2021

38 Overview Prostate Cancer. Diagnosis and Management | Guidance | NICE. Available from: https://www. nice.org.uk/ guidance/ngl31. Accessed April 9, 2021

39 Mottet N, Bellmunt J, Bolla M, et al. EAU-ESTRO-SIOG guidelines on prostate cancer. Part 1: screening, diagnosis, and local treatment with curative intent. Eur Urol 2017;71(4):618-629

40 FRAX. Available from. https://www.sheffield.ac.uk/FRAX/. Accessed April 9, 2021

41 James H II, Aleksic I, Bienz MN, et al. Comparison of fracture risk assessment tool score to bone mineral density for estimating fracture risk in patients with advanced prostate cancer on androgen deprivation therapy. Urology 2014;84(1):164-168

42 Smith MR, Halabi S, Ryan CJ, et al. Randomized controlled trial of early zoledronic acid in men with castration-sensitive prostate cancer and bone metastases: results of CALGB 90202 (alliance) J Clin Oncol 2014;32(11):1143-1150

43 Denosumab for the Prevention of Skeletal Complications in Metastatic Castration-Resistant Prostate Cancer. Comparison of Skeletal-Related Events and Symptomatic Skeletal Events. Annals of Oncology. Available from: https://www.annalsofoncology.org/ article/S0923-7534(19)31373-0/fulltext. Accessed April 9, 2021

44 Fizazi K, Carducci M, Smith M, et al. Denosumab versus zoledronic acid for treatment of bone metastases in men with castration-resistant prostate cancer: a randomised, double-blind study. Lancet 2011;377(9768):813-822

45 Smith MR, Saad F, Coleman R, et al. Denosumab and bone-metastasis-free survival in men with castration-resistant prostate cancer: results of a phase 3 , randomised, placebo-controlled trial. Lancet 2012;379(9810) :39-46

46 Smith MR, Egerdie B, Hernández Toriz N, et al. Denosumab HALT Prostate Cancer Study Group. Denosumab in men receiving androgen-deprivation therapy for prostate cancer. $\mathrm{N}$ Engl J Med 2009;361(8):745-755

47 Home. National Osteoporosis Foundation. Available from: https://www.nof.org/. Accessed April 9, 2021

48 Official Positions ISCD. Available from: https://www.iscd.org/ official-positions/. Accessed April 9, 2021

49 Boevé LMS, Hulshof MCC, Vis AN, et al. Effect on survival of androgen deprivation therapy alone compared to androgen deprivation therapy combined with concurrent radiation therapy to the prostate in patients with primary bone metastatic prostate cancer in a prospective randomised clinical trial: data from the HORRAD trial. Eur Urol 2019;75(3):410-418

50 Parker CC, James ND, Brawley CD, et al. Systemic Therapy for Advanced or Metastatic Prostate cancer: Evaluation of Drug Efficacy (STAMPEDE) investigators. Radiotherapy to the primary tumour for newly diagnosed, metastatic prostate cancer (STAMPEDE): a randomised controlled phase 3 trial. Lancet 2018;392(10162) :2353-2366

51 Burdett S, Boevé LM, Ingleby FC, et al. STOPCAP M1 Radiotherapy Collaborators. Prostate radiotherapy for metastatic hormone-sensitive prostate cancer: a STOPCAP systematic review and meta-analysis. Eur Urol 2019;76(1):115-124

52 Trabulsi EJ, Rumble RB, Jadvar H, et al. Optimum imaging strategies for advanced prostate cancer: ASCO guideline. J Clin Oncol 2020;38(17):1963-1996

53 National Comprehensive Cancer Network. Prostate Cancer (Version 2.2020). Available from: https://www.nccn.org/ professionals/physician_gls/pdf/prostate.pdf. Accessed April 9, 2021

54 Cornford P, Bellmunt J, Bolla M, et al. EAU-ESTRO-SIOG Guidelines on Prostate Cancer. Part II: treatment of relapsing, metastatic, and castration-resistant prostate cancer. Eur Urol 2017;71(4):630-642

55 Gillessen S, Attard G, Beer TM, et al. Management of patients with advanced prostate cancer: the report of the advanced prostate cancer consensus conference APCCC 2017. Eur Urol 2018;73(2):178-211

56 Giri VN, Knudsen KE, Kelly WK, et al. Implementation of germline testing for prostate cancer: Philadelphia prostate cancer consensus conference 2019. J Clin Oncol 2020; 38(24):2798-2811

57 de Bono J, Mateo J, Fizazi K, et al. Olaparib for metastatic castration-resistant prostate cancer. N Engl J Med 2020;382(22):2091-2102

58 Abida W, Patnaik A, Campbell D, et al; TRITON2 investigators. Rucaparib in men with metastatic castration-resistant prostate cancer harboring a BRCA1 BRCA2 gene alteration. J Clin Oncol 2020;38(32):3763-3772

59 Ramamurthy C, Handorf EA, Correa AF, Beck JR, Geynisman DM. Cost-effectiveness of abiraterone versus docetaxel in the treatment of metastatic hormone naïve prostate cancer. Urol Oncol 2019;37(10):688-695

60 Singh P, Agrawal T, Yadav S, Nayak B, Seth A, Dogra PN. A comparative study of the effects of medical versus surgical androgen deprivation therapy on health-related quality of life in patients with metastatic carcinoma prostate. Indian J Cancer 2018;55(2):148-153

61 Smith MR, Saad F, Hussain M, et al. ARASENS: a phase 3 trial of darolutamide in combination with docetaxel for men with metastatic hormone-sensitive prostate cancer (mHSPC) JCO 2018;36(6, suppl) :TPS383-TPS383

62 Attard G, Sydes MR, Mason MD, et al. Combining enzalutamide with abiraterone, prednisone, and androgen deprivation therapy in the STAMPEDE trial. Eur Urol 2014;66(5):799-802

63 A Phase III Study for Patients with Metastatic HormoneNaive Prostate Cancer-Full Text View-ClinicalTrials. Gov. Available from: https://clinicaltrials.gov/ct2/show/ NCT01957436. Accessed April 9, 2021 